\title{
Enhanced selectivity and search speed for method development using one-segment-per-component optimization strategies
}

\author{
Eva Tyteca ${ }^{\mathrm{a}, *}$, Kim Vanderlinden $^{\mathrm{a}}$, Maxime Favier ${ }^{\mathrm{b}}$, David Clicq ${ }^{\mathrm{b}}$, \\ Deirdre Cabooter ${ }^{\mathrm{c}}$, Gert Desmet ${ }^{\mathrm{a}}$ \\ a Department of Chemical Engineering (CHIS-IR), Vrije Universiteit Brussel, Belgium \\ b UCB Pharma, Belgium \\ c Department of Pharmaceutical Analysis, Katholieke Universiteit Leuven, Belgium
}

\section{A R T I C L E I N F O}

\section{Article history:}

Received 6 May 2014

Received in revised form 24 June 2014

Accepted 29 June 2014

Available online 6 July 2014

\section{Keywords:}

Method development

Retention modeling

LSS model

Non-linear retention model

Multi-segment gradients

Gradient optimization

\begin{abstract}
A B S T R A C T
Linear gradient programs are very frequently used in reversed phase liquid chromatography to enhance the selectivity compared to isocratic separations. Multi-linear gradient programs on the other hand are only scarcely used, despite their intrinsically larger separation power. Because the gradient-conformity of the latest generation of instruments has greatly improved, a renewed interest in more complex multisegment gradient liquid chromatography can be expected in the future, raising the need for better performing gradient design algorithms. We explored the possibilities of a new type of multi-segment gradient optimization algorithm, the so-called "one-segment-per-group-of-components" optimization strategy. In this gradient design strategy, the slope is adjusted after the elution of each individual component of the sample, letting the retention properties of the different analytes auto-guide the course of the gradient profile. Applying this method experimentally to four randomly selected test samples, the separation time could on average be reduced with about $40 \%$ compared to the best single linear gradient. Moreover, the newly proposed approach performed equally well or better than the multi-segment optimization mode of a commercial software package. Carrying out an extensive in silico study, the experimentally observed advantage could also be generalized over a statistically significant amount of different 10 and 20 component samples. In addition, the newly proposed gradient optimization approach enables much faster searches than the traditional multi-step gradient design methods.
\end{abstract}

(c) 2014 Elsevier B.V. All rights reserved.

\section{Introduction}

In reversed-phase liquid chromatography, selectivity can be obtained by varying many adjustable parameters: stationary phase chemistry, mobile phase composition, temperature, $\mathrm{pH}$ and of course by imposing a gradient mobile phase program [1-10]. Most gradient programs used in practice are simply linear. Multi-linear or multi-step gradient programs are only scarcely used, despite their intrinsically larger separation power. This lack of use is mainly due to the difficulty with which the (older generation) instruments can to comply to the imposed complex gradient program, as well as the lack of good search strategies to find the best multi-segment program among the innumerous possible combinations $[11,12]$.

\footnotetext{
* Corresponding author.

E-mail address: Eva.Tyteca@vub.ac.be (E. Tyteca).
}

Because the gradient-conformity of the latest generation of instruments has greatly improved, a renewed interest in more complex multi-segment gradient LC can be expected in the future, raising the need for better performing gradient design algorithms.

Many different stepwise or multi-isocratic gradient optimization methods have already been suggested in literature, such as overlapping separation range mapping [13], Monte-Carlo optimization [14], stationary phase optimized selectivity chromatography (SOSLC) [15], and stepwise elution chromatography through back calculation of the solvent concentration \%B from $R_{S}$ as a function of the retention factor (as a function of \%B) [16]. However, multilinear gradient optimization is mostly done in a trial-and-error fashion using simulation software such as DryLab [17,18]. This approach starts from the best linear gradient, inserting a number of node points, and subsequently dragging and dropping each node and evaluating the corresponding simulated chromatogram until a satisfactory separation is obtained. Another powerful approach is 


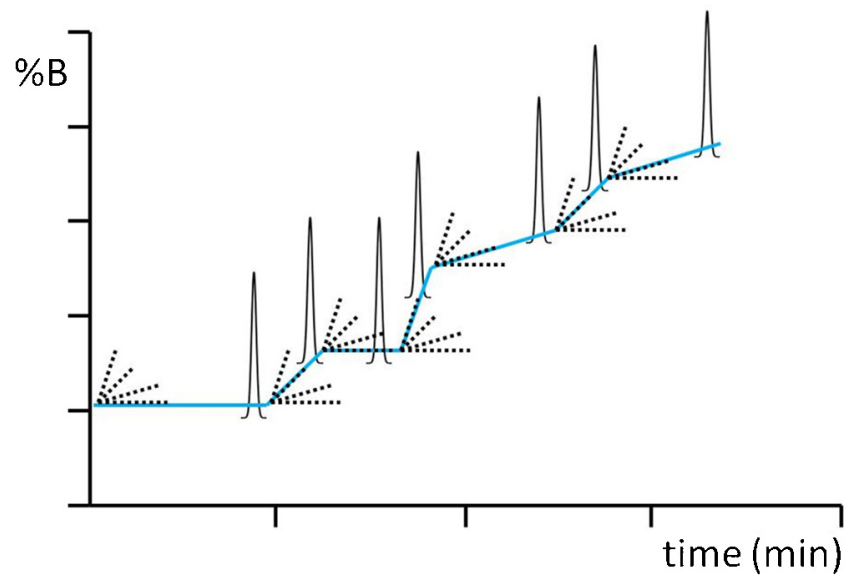

Fig. 1. "One-segment-per-component" multi-segment optimization approach using one segment for each eluting compound. The gradient steepness $\beta$ is optimized for every compound separately and each gradient segment is ended after the elution of the compound.

Chromsword. This software combines chromatogram simulation and fast Monte-Carlo optimization to optimize multi-linear gradients [19]. In 2006, Nikitas and coworkers reported on the use of Genetic Algorithms to optimize multi-linear gradients [20,21]. However, this optimization approach requires a priori selection of initial parameters such as the number of generations, the population size and the probability of mutation [20]. Concha-Herrara and coworkers investigated the benefit of including more and more segments using a time consuming grid search as optimization strategy. To guide this search the concept of limiting peak purity was used [12]. The authors reported that gradients including more than four segments did not enhance the separation.

The present study has been set up to explore the possibilities of a new type of multi-segment gradient optimization algorithm, the so-called one-segment-per-component optimization strategy. In this approach, the slope of the gradient program is allowed to be adjusted after the elution of each individual component of the sample (Fig. 1). Doing so, a very high degree of flexibility is given to the gradient program, which in turn should increase the probability of finding the most optimal gradient program in terms of speed and/or robustness. In addition, the gradient optimization process is guided by the retention properties of the components (which are assumed to be known via a set of independent measurements) such that no time is wasted to exploring parts of the solution parameter space where anyhow no good selectivity can be expected.

The method assumes that the retention parameters of the individual compounds are accurately known and runs as follows. First, different slopes and $\phi_{0}$-values for the first segment are considered, and the known retention properties are used to calculate which compound will elute first. Subsequently, different slopes are considered for the second segment (whose starting point is determined by the elution of the first component), for each of the different possible slopes in the first segment. Again, it is checked which one of the remaining compounds will be eluting first from this second segment. This process is repeated until all components have eluted (using one gradient segment per component). As schematically depicted in Fig. 1, the total number of combinations that needs to be searched in this way corresponds to a search tree with $x^{y}$ branches (wherein $x=$ number of compounds and $y=$ number of considered gradient slopes). During the search, not all the branches have to explored till the very end because a given branch can be considered as non-optimal as soon as one of the peak pairs elutes with a resolution below the minimal threshold corresponding to the best critical resolution $R_{s, \text { crit }}$ that was already obtained during the search (in order to never consider a sequence that is worse than the already considered sequences). This search strategy was implemented via a self-written MATLAB ${ }^{\circledR}$-routine.

The present study contains both a numerical as well as an experimental comparison of the newly proposed one-segmentper-component optimization strategy with the conventional grid search for single and multi-step gradients. The numerical study was conducted to cover a statistically relevant number of different samples (463 in the present case). The experimental comparison was run to illustrate how the approach would work for a number of typical practical separation problems.

\section{Material and methods}

\subsection{Numerical methods}

\subsubsection{Retention modeling}

In the experimental part, the non-linear empirical retention model proposed by Neue and Kuss [22] was used to describe the curved relationship between the natural logarithm of the retention factor $\mathrm{k}$ and the fraction of organic modifier in the mobile phase $\phi$ :

$\ln (k)=\ln \left(k_{w}\right)+2 \ln \left(1+S_{2} \varphi\right)-\frac{S_{1} \varphi}{1+S_{2} \varphi}$

where $k_{w}$ is the retention factor in pure water, $S_{1}$ is the slope ( $\sim$ solvent strength parameter in the LSS-model from Snyder and Dolan $[23,24]$ ) and $S_{2}$ is the curvature coefficient. If $S_{2}$ is equal to zero Eq. (1) reduces to the conventional LSS-model.

The three-parameter Neue and Kuss-model was preferred over the more conventional (and also empirical) two-parameter LSSmodel, because the former is inherently more accurate (because of the third parameter allowing curvature in the $\ln (k)$ vs. $\varphi$ relation), especially at higher percentages of organic modifier [22]. As the one-segment-per-component method intrinsically allows to put the peaks in the chromatograms more closer to each other than the conventional gradient methods, it should be evident that any gain in prediction accuracy, even though it is only by $1 \%$, can already be very significant. The predictions obtained with the LSSmodel were simply not good enough to confidently calculate and experimentally verify the best possible gradient program.

To predict the elution times of the individual compounds, the fundamental gradient equation [25] was extended for multisegmented gradients, taking into account the instrument dwell time $t_{D}$. For an $n$-segmented gradient this becomes:

$t_{0}=\int_{0}^{t_{D}} \frac{\mathrm{d} t_{S}}{k\left(\varphi_{0,1}\right)}+\sum_{i=1}^{n-1} \int_{\varphi_{0, i}=\varphi_{e, i-1}}^{\varphi_{e, i}} \frac{1}{\beta_{i}} \frac{\mathrm{d} \varphi}{k(\varphi)}+\int_{\varphi_{0, n}=\varphi_{e, n-1}}^{\varphi_{\text {elution }}} \frac{1}{\beta_{n}} \frac{\mathrm{d} \varphi}{k(\varphi)}$

where $\beta$ is the gradient steepness defined as $\beta=\left(\phi_{e}-\phi_{0}\right) / t_{G}$.

For a simple linear gradient, the summation in the second term on the right hand side of in Eq. (2) disappears. Solving Eq. (2) gives an expression for $\phi_{\text {elution }}$ from which an expression for $k_{\text {eff }}$ (defined as the reduced retention time per column hold-up volume: $\left.k_{\text {eff }}=\left(t_{R}-t_{0}\right) / t_{0}\right)$ can be easily found via the following relation:

$\phi_{\text {elution }}=\phi_{0}+\beta\left(t_{R}-t_{0}-t_{D}\right) \Leftrightarrow k_{\text {eff }}=\frac{t_{R}-t_{0}}{t_{0}}=\frac{t_{D}}{t_{0}}+\frac{\phi_{\text {elution }}-\phi_{0}}{\beta t_{0}}$ 
Implementing Eq. (1) for the compound eluting with the first segment, the expression for the effective gradient retention factor $k_{\text {eff }}$ becomes:

$k_{\mathrm{eff}, 1}=\frac{t_{D}}{t_{0}}+\frac{\begin{array}{c}\left(\phi_{0,1}+\left(\left(1+S_{2} \phi_{0,1}\right) / S_{1}\right) \ln \left(\beta_{1} k_{w}^{\prime} S_{1}\left(t_{0}-t_{D} / k_{0}\right) \exp \left(-S_{1} \phi_{0,1} /\left(1+S_{2} \phi_{0,1}\right)\right)\right)\right) /\left(1-S_{2}\left(1+S_{2} \phi_{0,1}\right) / S_{1}\right. \\ \left.\left.k_{w}^{\prime} S_{1}\left(t_{0}-t_{D} / k_{0}\right) \exp \left(-S_{1} \phi_{0,1} /\left(1+S_{2} \phi_{0,1}\right)\right)\right)\right)-\phi_{0,1}\end{array}}{\beta_{1} t_{0}}$

For components eluting during one of the following segments $(n>1), k_{\mathrm{eff}}$ is given by

$$
\begin{aligned}
( & \phi_{0,1}+\left(\left(1+S_{2} \phi_{0,1}\right) / S_{1}\right) \ln \left(\beta_{n} k_{w}^{\prime} S_{1}\left(t_{0}-t_{D} / k_{0}\right) \exp \left(-S_{1} \phi_{0,1} /\left(1+S_{2} \phi_{0,1}\right)\right)+\sum_{i=1}^{n-1}\left(\beta_{n} / \beta_{i+1}-\beta_{n} / \beta_{i}\right)\right. \\
& \left.\left.\times \exp \left(S_{1} \phi_{e, i} /\left(1+S_{2} \phi_{e, i}\right)\right) \exp \left(-S_{1} \phi_{0,1} /\left(1+S_{2} \phi_{0,1}\right)\right)+\beta_{n} / \beta_{1} s\right)\right) /\left(1-\left(S_{2}\left(1+S_{2} \phi_{0,1}\right) / S_{1}\right) \ln \left(\beta_{n} k_{w}^{\prime} S_{1}\left(t_{0}-t_{D} / k_{0}\right)\right.\right. \\
& \times \exp \left(-S_{1} \phi_{0,1} /\left(1+S_{2} \phi_{0,1}\right)\right)+\sum_{i=1}^{n-1}\left(\beta_{n} / \beta_{i+1}-\beta_{n} / \beta_{i}\right) \exp \left(S_{1} \phi_{e, i} /\left(1+S_{2} \phi_{e, i}\right)\right) \\
k_{\mathrm{eff}, n}=\frac{t_{D}}{t_{0}}+\frac{}{\beta_{n} t_{0}} & \left.\left.\times \exp \left(-S_{1} \phi_{0,1} /\left(1+S_{2} \phi_{0,1}\right)\right)+\beta_{n} / \beta_{1}\right)\right)-\phi_{0,1}
\end{aligned}
$$

The retention parameters of the compounds in the experimental part of the present study were determined by performing three linear gradient runs with different gradient times $t_{G}$, starting and/or final concentration of organic modifier (5 to 95\%B in 5 and $10 \mathrm{~min}$ and 20 to $80 \% \mathrm{~B}$ in $5 \mathrm{~min}$ ). The $k_{w^{\prime}}{ }^{\prime}, S_{1}$ - and $S_{2}$-parameters were obtained by solving these three equations (Eq. (4)) using the lsqcurvefit routine in $\mathrm{MATLAB}^{\odot}$. It should be noted that not $\ln \left(k_{\text {eff }}\right)$ values but $k_{\text {eff }}$ values were fitted to obtain the model parameters. This was done to reduce the problems of least squares fitting in semi-log scale (small errors in the logarithmic scale being much larger after back transformation).

In the numerical part of this study, $S_{2}$ was set equal to zero for the sake of simplicity (when $S_{2}=0$ the Neue and Kuss-model reduces to the LSS-model), because it can be inferred that the nature of the retention model will have no impact on the outcome of the comparison study. Moreover, the use of the pure LSS-model enabled us to consider very realistic $k_{w}$ - and $S_{1}$-ranges, as the $k_{w}$ - and $S$-values needed in the LSS-model have already been reported in literature for many compounds, whereas this is not yet the case for the $k_{w}{ }^{\prime}$ , $S_{1}$ - and $S_{2}$-parameters from Eq. (1). It would therefore be more difficult to find a set of $k_{w}{ }^{\prime}-, S_{1}$ - and $S_{2}$-ranges that represent reality.

\subsubsection{Gradient optimization strategies}

2.1.2.1. Linear gradients. The best possible linear gradient parameters $\left(\phi_{0}, \phi_{\mathrm{e}}\right.$ and $\left.t_{G}\right)$ were determined via a conventional grid search [26] using Eq. (4), implemented via an in-home written MATLAB ${ }^{\odot}$ code. In this code, the retention of each compound in the sample was calculated for each component in the sample using the measured values for $k_{w}{ }^{\prime}, S_{1}$ and $S_{2}$ for each component in the sample, for each physically possible combination of $\phi_{0}$ (going from 0.05 to 0.95 with step size of 0.01 ) and $\beta$ ( $\beta$ going from 0.001 to 0.5 , i.e., $\ln (\beta)$ going from -6.9 to -0.69 with step size of 0.07 ) to find the combination giving the best separation in the shortest possible analysis time. Solutions for which the last component eluted after $k_{\text {last, } \max }=25$ were rejected to prevent finding solutions with unnecessarily long analysis times. If the experimentally obtained chromatogram (=partially optimized chromatogram) showed more than baseline resolution (resulting in a time gap between the critical pairs), the linear gradient was manually fine-tuned by shortening the gradient time $t_{G}$ (while keeping $\phi_{0}$ and $\phi_{e}$ the same) to further speed up the method, until baseline separation was lost.

2.1.2.2. "Traditional" multi-segment gradients. The best "traditional" multi-segment gradient profile (determined by a starting composition $\phi_{0}$ and a value for $\beta$ and $t_{G}$ for each segment) was determined via a similar grid search. Based on our own findings and these of Concha-Herrara et al. [12], only 4-segment gradients were considered as these give the best compromise between the achievable selectivity (in gradient elution this is the ratio between the apparent gradient retention factors $k_{\mathrm{eff}, 1} / k_{\mathrm{eff}, 2}$ [16-27]) and the required search time. The grid search was conducted considering different starting concentrations \%B between 5 and 95\% (step size of $0.5 \%$ ) and a number of $\beta$ - and $t_{G}$-values for each of the 4 segments ( $\beta$ going from 0.001 to 0.2 , corresponding to 0.1 to $20 \% \mathrm{~B} / \mathrm{min}$, i.e., $\ln (\beta)$ between -6.9 and -0.70 and $t_{G} / t_{0}$ between 1 and 12$)$. The best possible grid was used to compare with the newly proposed "one-segment-per-component" search. The details of the number of investigated combinations of $\beta$ - and $t_{G}$-values used in the numerical comparison study are given in Table 1 .

2.1.2.3. "One-segment-per-component" gradients. The newly proposed "one-segment-per-component" gradients were also optimized using a similar grid search approach. However, the "one-segment-per-component" search only involves the optimization of the $\beta$-values of each segment (search for $\beta$ going from 0.001 to 0.2 , see caption of Table 1 for specific values) and does not have to consider different $t_{G}$-values, because the length of each segment $t_{G, n}$ is automatically ended by the elution of the next eluting peak. As a consequence, the length of each segment $t_{G, n}$ is no longer an explicit search variable, as it is determined by:

$t_{G, n}=t_{R, n}-t_{D}-t_{0}-\sum_{i=1}^{n-1} t_{G, i}$

where $t_{R, n}$ is the retention time of the $n$th eluting peak. For the experimental samples, the considered $\beta$ - and $t_{G}$-values used in the search grid were the same as in sample set 1 of the numerical study. When the obtained simulated chromatogram showed a $R_{s, \text { crit }}>1.6$, the optimization program was run again using a smaller $k_{\text {last,max }}$ (i.e., the maximal $k$-value for the last eluting compound) until full resolution was no longer obtained. Although the method (and more specifically the nature of Eq. (5)) easily allows the incorporation of negative gradient slopes, this possibility was excluded as it was our experience that the potential gain in selectivity usually does not compensate for the additional peak broadening effect originating from the negative gradient.

A limitation of the "one-segment-per-component" approach is that it can lead to gradient profiles that are too complex to be practically feasible when the samples become too complex ( $\geq 20$ compounds). Moreover, the calculation time would increase limiting the flexibility of the approach. For such samples, a modification was made by including two compounds per segment instead of only one, in order to keep the maximum number of 
Table 1

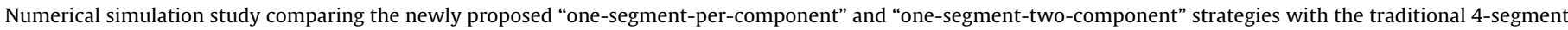

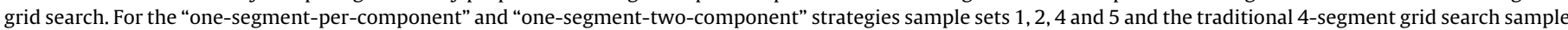

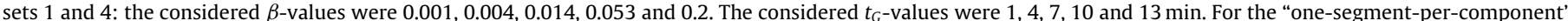

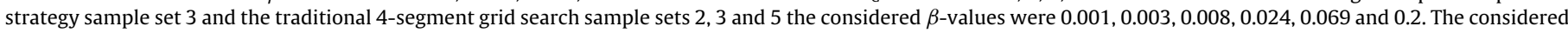
$t_{G}$-values were $1,3.5,6,8.5,11$ and $13.5 \mathrm{~min}$.

\begin{tabular}{|c|c|c|c|c|c|c|c|}
\hline Sample set & $\begin{array}{l}\text { Nr. of } \\
\text { compounds }\end{array}$ & Search strategy & $\begin{array}{l}\text { Number of } \\
\text { search points }\end{array}$ & $\begin{array}{l}\text { Average search } \\
\text { time per sample (s) }\end{array}$ & $k_{\text {last,average }}$ & Wins & Ex-aequo \\
\hline 1 & 10 & $\begin{array}{l}\text { One-segment-per-component } \\
\text { Four-segment grid }\end{array}$ & $\begin{array}{r}95 \times 5^{10} \\
95 \times 5^{4} \times 5^{4}\end{array}$ & $\begin{array}{r}86 \\
205\end{array}$ & $\begin{array}{r}9.95 \\
11.27\end{array}$ & $\begin{array}{r}118 \\
7\end{array}$ & 35 \\
\hline 2 & 10 & $\begin{array}{l}\text { One-segment-per-component } \\
\text { Four-segment grid }\end{array}$ & $\begin{array}{r}95 \times 5^{10} \\
95 \times 6^{4} \times 6^{4}\end{array}$ & $\begin{array}{r}138 \\
1465\end{array}$ & $\begin{array}{r}10.60 \\
9.50\end{array}$ & $\begin{array}{l}18 \\
36\end{array}$ & 20 \\
\hline 3 & 10 & $\begin{array}{l}\text { One-segment-per-two-components } \\
\text { Four-segment grid }\end{array}$ & $\begin{array}{r}95 \times 6^{10} \\
95 \times 6^{4} \times 6^{4}\end{array}$ & $\begin{array}{l}1216 \\
1410\end{array}$ & $\begin{array}{r}9.10 \\
10.02\end{array}$ & $\begin{array}{r}24 \\
4\end{array}$ & 6 \\
\hline 4 & 20 & $\begin{array}{l}\text { One-segment-per-two-components } \\
\text { Four-segment grid }\end{array}$ & $\begin{array}{r}95 \times 5^{10} \\
95 \times 5^{4} \times 5^{4}\end{array}$ & $\begin{array}{r}80 \\
373\end{array}$ & $\begin{array}{l}12.57 \\
13.95\end{array}$ & $\begin{array}{r}107 \\
4\end{array}$ & 49 \\
\hline 5 & 20 & $\begin{array}{l}\text { One-segment-per-two-components } \\
\text { Four-segment grid }\end{array}$ & $\begin{array}{r}95 \times 5^{10} \\
95 \times 6^{4} \times 6^{4}\end{array}$ & $\begin{array}{r}66 \\
3955\end{array}$ & $\begin{array}{l}13.57 \\
13.51\end{array}$ & $\begin{array}{r}9 \\
12\end{array}$ & 14 \\
\hline
\end{tabular}

segments limited. This approach is referred to further on as the "one-segment-per-two-components" approach.

2.1.2.4. Optimization goal. In each case, the optimization goal of the searches was expressed via a chromatographic response function (CRF) [28]. Since the retention properties of the analytes are in any case known, the most appropriate CRF simply corresponds to the resolution of the critical pair $R_{s, \text { crit }}$ of the final chromatogram. To speed up the calculations in the numerical comparison part of the study, only the retention times of the compounds were taken into account. In this case, the expression for $R_{s, \text { crit }}$ reduces to:

$\mathrm{CRF}=\min \left(1.6, R_{s, \text { crit }}\right) \quad$ with $\quad R_{s, \text { crit }}=\frac{\sqrt{N}}{4} \frac{\Delta k_{\text {crit }}}{1+k_{\text {elution,crit }}}$

where $\Delta k_{\text {crit }}$ is the difference in $k$ between the peaks of the critical pair and $k_{\text {elution,crit }}$ the retention factor at elution. The value of $N$ (defined in Eq. (7) as the number of theoretical plates that would be observed under gradient elution conditions, see Eq. (2) in Neue [29] for definition of $N$ ) was put arbitrarily at $N=20,000$ in the numerical comparison part of the study. The actually employed value of $N$ is however totally irrelevant in the above procedure, because the same $N$ value was taken for every compound, such that the obtained chromatograms were anyhow only judged on the selectivity among the different components.

Experimentally, we adopted the same procedure as Drylab, i.e., filling in the plate number obtained by simply estimating $\mathrm{N}$ from a simple van Deemter-equation (roughly leading $N=20,000$ in our case, where we have put $A=1.2, B=3.1$ and $C=0.15$ ). The resolution we obtained in the simulated chromatograms were in very good agreement with the experimentally observed resolution.

In both the numerical and experimental part of the study, the maximization of $R_{s, \text { crit }}$, was stopped when it reached a maximal value of 1.6, i.e., corresponding to a full baseline separation when considering the case of equally high peaks. This upper limit was imposed to prevent favoring solutions with an unnecessarily large spacing between the peaks. To find the gradient conditions giving the best separation in the shortest possible time, the CRF furthermore also included a time component, implemented via a conditional statement ("if-then-else" construct). As long as the CRF increases (while $R_{s, \text { crit }}<1.6$ and $t_{R \text {,last }}<t_{R, \max }$ ) the newly proposed gradient program is retained, irrespective of $t_{R \text {,last }}$. On the other hand, once full resolution is obtained $\left(R_{s, \text { crit }}=1.6\right)$, only conditions that have an $t_{R \text {, last }}$ that is smaller than the one already obtained are retained.

During the searches conducted for the 'one-segment-percomponent'-approach, the local $\left(R_{S, n}\right)$ between the compound eluting in the gradient segment under optimization and the one eluting in the previous segment was always considered as well. Whenever this local $R_{S, n}$ was insufficient $\left(R_{S, n}<1.6\right)$, the sequence is stopped, and the calculation restarts at the first compound. This allowed speeding up the search, because in this way not all the different branches of the search tree need to be explored till the end. This is different from the traditional 4-segment grid search, where the search can only be stopped after each of the four segments.

\subsection{Experimental}

\subsubsection{Sample 1: 15 Tar oil degradation products}

The composition of the tar oil degradation products (WWP) mixture was based on a study performed by Cabooter et al. [30] and consisted of (1) quinoline, (2) fluorene, (3) benzofuran, (4) 2-naphthol, (5) indene, (6) 1-indanon, (7) 9-hydroxyfluorene, (8) dibenzofuran, (9) 2-hydroxyquinoline, (10) 1-benzothiophene, (11) dibenzothiophene sulfone, (12) 2-naphthoic acid, (13) acenapthene, (14) carbazole and (15) indane. All compounds except benzothiophene were purchased from Sigma-Aldrich, Bornem, Belgium. Benzothiophene was purchased from VWR, Leuven, Belgium. All components were dissolved to a final concentration of $100 \mathrm{ppm}$ in $5 / 95 \mathrm{vol} \% / \mathrm{vol} \% \mathrm{H}_{2} \mathrm{O} / \mathrm{ACN}$. The mobile phase consisted of $(\mathrm{A}) \mathrm{H}_{2} \mathrm{O}$ with $10 \mathrm{mM}$ ammonium acetate, adjusted to $\mathrm{pH} 6.8$ with ammonium hydroxide and (B) ACN. ACN was purchased from Biosolve. The separation was performed on an Agilent Infinity 1290 system with a dwell volume of $112 \mu \mathrm{L}$ using a $100 \mathrm{~mm}$ Waters Acquity UPLC BEH phenyl column $(2.1 \times 100 \mathrm{~mm}, 1.7 \mu \mathrm{m})$. The injection volume was $1 \mu \mathrm{L}$. The flow rate was $0.6 \mathrm{~mL} / \mathrm{min}$ and the temperature was $23^{\circ} \mathrm{C}$. The column dead time was $0.35 \mathrm{~min}$. The components were detected at $210 \mathrm{~nm}$ (sample rate of $80 \mathrm{~Hz}$ ). Scouting runs were 5-95\%B in 5 and $10 \mathrm{~min}$ and $20-80 \% \mathrm{~B}$ in $5 \mathrm{~min}$.

\subsubsection{Sample 2: API and 13 impurities}

The pharmaceutical mixture provided by UCB Pharma (Braine l'Alleud, Belgium) consisted of 14 closely related compounds (API and 13 impurities). The identities of the compounds are confidential and are therefore not listed. The separation was performed on a Waters UPLC system with a dwell volume of $72 \mu \mathrm{L}$ using a Waters Acquity $\mathrm{CSH} \mathrm{C}_{18}$ column $(2.1 \times 100 \mathrm{~mm}, 1.7 \mu \mathrm{m})$. The column temperature was set $40^{\circ} \mathrm{C}$. The flow rate was $0.6 \mathrm{~mL} / \mathrm{min}$. The column dead time was $0.61 \mathrm{~min}$. The mobile phase consisted of (A) a $50 \mathrm{mM}$ ammonium acetate buffer with $0.04 \%$ formic acid and (B) ACN with $0.04 \%$ formic acid. All compounds were dissolved to a final concentration of $500 \mathrm{ppm}$ and the injection volume was $2 \mu \mathrm{L}$. Chromatograms were measured at $210 \mathrm{~nm}$ (sample rate of $80 \mathrm{~Hz}$ ). 
Scouting runs were 5-95\%B in 5, 10 and 15 min and 20-80\%B in 5 and $10 \mathrm{~min}$.

\subsubsection{Sample 3 and 4: 14 and 16 drugs commonly found in waste water}

The 16 component drugs sample consisted of (1) ethinylestradiol, (2) sulfamethoxazole, (3) ibuprofen, (4) ticlopidine, (5) testosterone, (6) oxazepam, (7) estrone, (8) carbamazepine, (9) lorazepam, (10) paracetamol, (11) diclofenac, (12) caffeine, (13) zolpidem, (14) trimethoprim, (15) progesterone and (16) $\beta$ estradiol. For the 14 component sample, component 15 and 16 were left out. Components 1, 5-7, 11 and 14-16 were dissolved in EtOH (Sigma-Aldrich), components 2, 3, 8 and 9 in ACN and components 4, 10, 12 and 13 in $\mathrm{H}_{2} \mathrm{O}$ (British Pharmacopoeia, 2012). All compounds were dissolved to a final concentration of $50 \mathrm{ppm}$ in EtOH (Sigma-Aldrich), except for component 5 and 15 which had a final concentration of $200 \mathrm{ppm}$. The mobile phase used for the separation of these mixtures consisted of (A) a $10 \mathrm{mM}$ ammonium formate buffer adjusted to $\mathrm{pH} 3$ with formic acid and (B) ACN. The flow rate was $0.4 \mathrm{~mL} / \mathrm{min}$. The column dead time was $0.53 \mathrm{~min}$. The injection volume was $1 \mu \mathrm{L}$. The separation was performed on a Agilent Infinity 1290 system with a dwell volume of $112 \mu \mathrm{L}$ using a Waters Acquity BEH $\mathrm{C}_{18}$ column $(2.1 \times 100 \mathrm{~mm}, 1.7 \mu \mathrm{m})$. Column temperature was set $25^{\circ} \mathrm{C}$. Chromatograms were measured at $210 \mathrm{~nm}$ (sample rate of $80 \mathrm{~Hz}$ ). Scouting runs were 5-95\%B in 5 and $10 \mathrm{~min}$ and $20-80 \% \mathrm{~B}$ in $5 \mathrm{~min}$. For components 1 and 7 two extra scouting runs $(10-65 \% \mathrm{~B}$ in $5 \mathrm{~min}$ and $30-60 \% \mathrm{~B}$ in $5 \mathrm{~min}$ ) were performed to determine the model parameters.

\section{Results and discussion}

\subsection{Results of numerical study}

In-silico samples with different complexity, i.e., containing either 10 or 20 compounds, were composed by randomly attributing each component a physically possible $k_{w^{-}}$and $S$-value taken from eight different ranges (Table S-1). This was done for 160 samples per sample complexity, in groups of 20 per considered $k_{w}$-and $S_{1}$-range (as already mentioned, $S_{2}=0$ for all compounds was taken for the sake of simplicity). The optimal gradient program was subsequently determined following the procedures described in Section 2.1.2 for the traditional 4-segment gradient and the presently proposed "one-segment-per-component" (10 component samples) and "one-segment-per-two-components" (20 component samples) gradient optimization algorithms.

To distinguish between the required search time and the possibility to obtain the best selectivity as the performance criteria, the total search time of the methods has been varied by changing the number of considered $\beta$ - and $t_{G}$-values. The different sample sets with their corresponding number of search points are given in Table 1. Sample sets 1 to 3 included the 10 -compound samples and sample sets 4 and 5 included the 20-compound samples. For all sample sets the number of possible starting combinations $\% \mathrm{~B}_{\text {start }}$ was 95 (from $1 \%$ to $95 \%$ with step size of $1 \%$ ). In sample set 1 , five different slopes $\beta$ were considered for each segment, hence giving $95 \times 5^{10}$ combinations for the "one-segment-per-component" search in case of the 10 component samples. For the traditional 4-segment grid search five different lengths and five different slopes were considered per segment, hence a total of $95 \times 5^{4} \times 5^{4}$ combinations needed to be considered in the search. Although the number of considered combinations is lower for the traditional 4-segment grid search, the search times are larger because the number of combinations that is calculated during the search is higher. As mentioned in Section 2.1.2, the one-segment-percomponent" approach allows to stop calculating a given sequence after each component (or each pair of compounds in case of the one-segment-two-components) when the local $R_{s, n}$ between this compound and the compound before is insufficient, while the traditional 4-segment grid search can only do this after each of the four segments. In sample sets 2, 3 and 5, the search time of the traditional grid search was increased by considering six instead of only 5 different slopes and segment lengths. In sample set 3 the "one-segment-per-component" strategy also included six different slopes, hence also increasing its search time.

Next to the calculation time also the analysis time ( $\left.k_{\text {last }}\right)$ is reported for every sample set in Table 1. A win-loss comparison was made by comparing the retention factor of the last compound $k_{\text {last }}$. A "win" for strategy 1 was reported when $k_{\text {last,strategy } 1}<k_{\text {last,strategy } 2}-0.5$. A difference of 0.5 in $k_{\text {last }}$ was considered to be a significant difference in order to get representative results. When the difference in $k_{\text {last }}$ was less than 0.5 an ex-aequo between the two strategies was reported. For the 10 component samples, the "one-segment-per-component" nearly always wins even when it is allotted a smaller search time than the 4-segment grid search (118 wins vs. 7 wins for the 4 -segment grid search, see sample set 1 ). The 4 -segment grid only manages to slightly outperform the "one-segment-per-component" strategy when it is allotted a 10 times larger search (sample set 2). When the time allotted to the "one-segment-per-component" search is then again increased to the same level (sample set 3 ), the latter again wins convincingly. In sample sets 4 and 5 , a similar conclusion can be drawn for the "one-segment-per-two-components"-strategy applied to 20 component samples. Here, a 50 times larger search time is needed before the traditional 4-segment grid produces a similar number of wins as the "one-segment-per -two-components"strategy (sample set 5). When the traditional 4-segment grid is only allotted a 5 times larger search time (sample set 4 ), the latter only gets 4 wins vs. 107 wins for the "one-segment-per -twocomponents"-strategy. For each comparison a new set of samples was generated, hence explaining the difference in search times for the "one-segment-per-component" and the "one-segment-pertwo-components" strategies, respectively, among sample sets 1-3 and among sample set 4-5 in Table 1.

When comparing both methods for a similar number of search points (data set 3 ), the time gain is $9 \mathrm{~min}$ vs. $10 \mathrm{~min}$. Although some of the solutions obtained for the 4-segment procedure might have a slightly higher resolution than those obtained with the 1comp-per-segment (although also the opposite occurs), and higher resolution can in theory be traded for a shorter analysis time, this time gain is much larger than can be explained by the residual differences in resolution.

This over-all better performance can be understood as follows. In the traditional multi-step gradient approach, the breakpoints between the segments are introduced at random points. The "onesegment-per-component" approach (Section 2.1.2) on the other hand uses the retention characteristics of the sample compounds to introduce a breakpoint time after the elution of each component. As a consequence, the trajectory of the gradient program is auto-guided by the elution properties of the sample compounds, such that no time is wasted to searching in areas of the parameter space where there is anyhow no chance of finding a good solution as is inevitably the case in the traditional multi-step gradient approach. Furthermore, the one-segment-per-component" approach also allows to stop calculating a given sequence after each component (or each pair of compounds in case of the onesegment-two-components) when the local resolution $R_{s, n}$ between this compound and the compound eluting in the previous segment is insufficient. This provides an additional gain in search time vs. number of search points (Table 1 ).

Moreover, because the present approach uses the information on the retention properties of the compounds, the search for the 

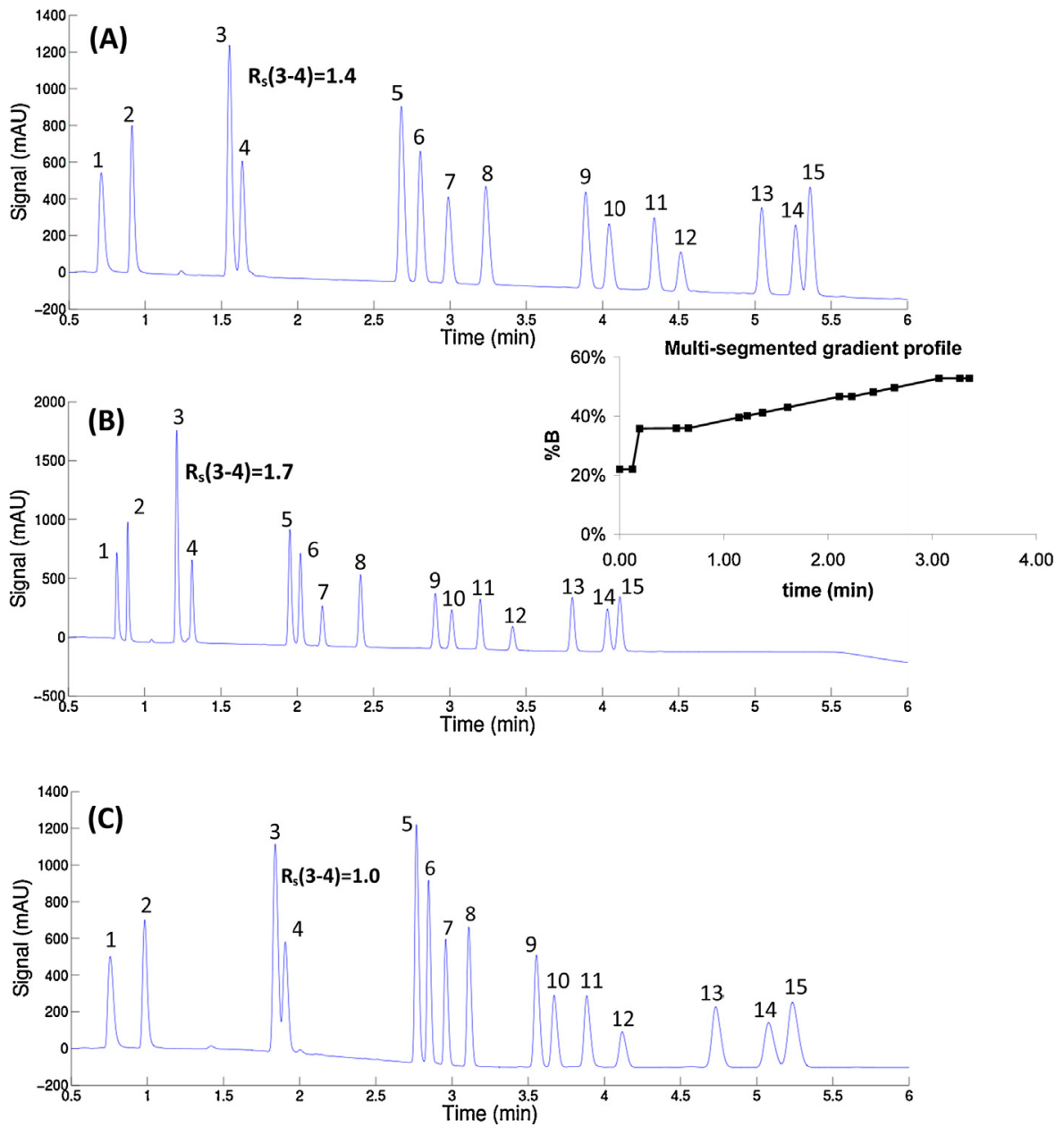

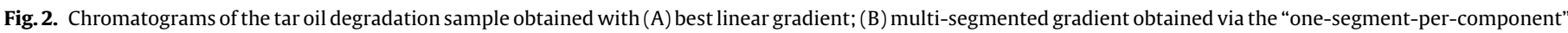
approach; (C) 3-segment gradient obtained with the fully optimized method development mode of a commercial software package.

best gradient program is automatically directed in the right direction, in contrast to a traditional grid search, in which the solution space is scanned in a uniform way without taking into account the retention properties of the compounds. The latter will result in the unnecessary calculation of irrelevant and non-optimal gradients conditions.

\subsection{Experimental results}

To validate the "one-segment-per-component" approach experimentally, it has also been applied to four randomly selected examples. In each case, the newly proposed multi-segment optimization strategy allowed to speed up the separation compared to the best linear gradient. Moreover, it performed equally well or better than the multi-segment optimization mode of Chromsword software.

\subsubsection{Sample 1: 15 Tar oil degradation products}

The first sample includes 15 tar oil degradation products. With the "one-segment-per-component" optimization strategy a 15segment gradient was found including a small isocratic step at the beginning. The one-segment-per-component gradient is in fact a 7 -segment gradient as many consecutive gradient segments have (almost) the same gradient slope. The analysis time of the 15 tar oil degradation products could be reduced by $24 \%$ compared to the best linear gradient ( $24.0 \% \mathrm{~B}$ to $59.0 \% \mathrm{~B}$ in $5.60 \mathrm{~min}$ ) proposed by Cabooter et al. [30] (Fig. 2A and B). Using the Neue-Kuss model described by
Eq. (1), the mean deviation between the predicted and the observed $k$-values for the obtained "one-segment-per-component" gradient was $2.3 \%$ (Table 2). The sample was also separated using the fully optimized method development function of the commercial software package software. After a first screening step (screening mode) the separation was optimized using the fine optimization mode, resulting in a three-segmented gradient (Table S-2). The corresponding separation has roughly the same analysis time as with the best linear gradient and thus much slower than with the

Table 2

Predicted and experimental retention times for Sample 1 (15 tar oil degradation products) obtained via the "one-segment-per-component" approach.

\begin{tabular}{lll}
\hline$t_{R, \text { predicted }}$ & $t_{R, \exp }$ & Deviation (\%) \\
\hline 0.86 & 0.80 & -7.1 \\
0.93 & 0.89 & -4.6 \\
1.28 & 1.22 & -4.9 \\
1.40 & 1.30 & -7.1 \\
1.88 & 1.90 & 1.0 \\
1.96 & 1.98 & 0.9 \\
2.11 & 2.12 & 0.5 \\
2.35 & 2.36 & 0.4 \\
2.85 & 2.84 & -0.2 \\
2.97 & 2.95 & -0.6 \\
3.17 & 3.13 & -1.4 \\
3.38 & 3.34 & -1.0 \\
3.80 & 3.74 & -1.7 \\
4.00 & 3.96 & -1.1 \\
4.09 & 4.04 & -1.3 \\
\hline
\end{tabular}




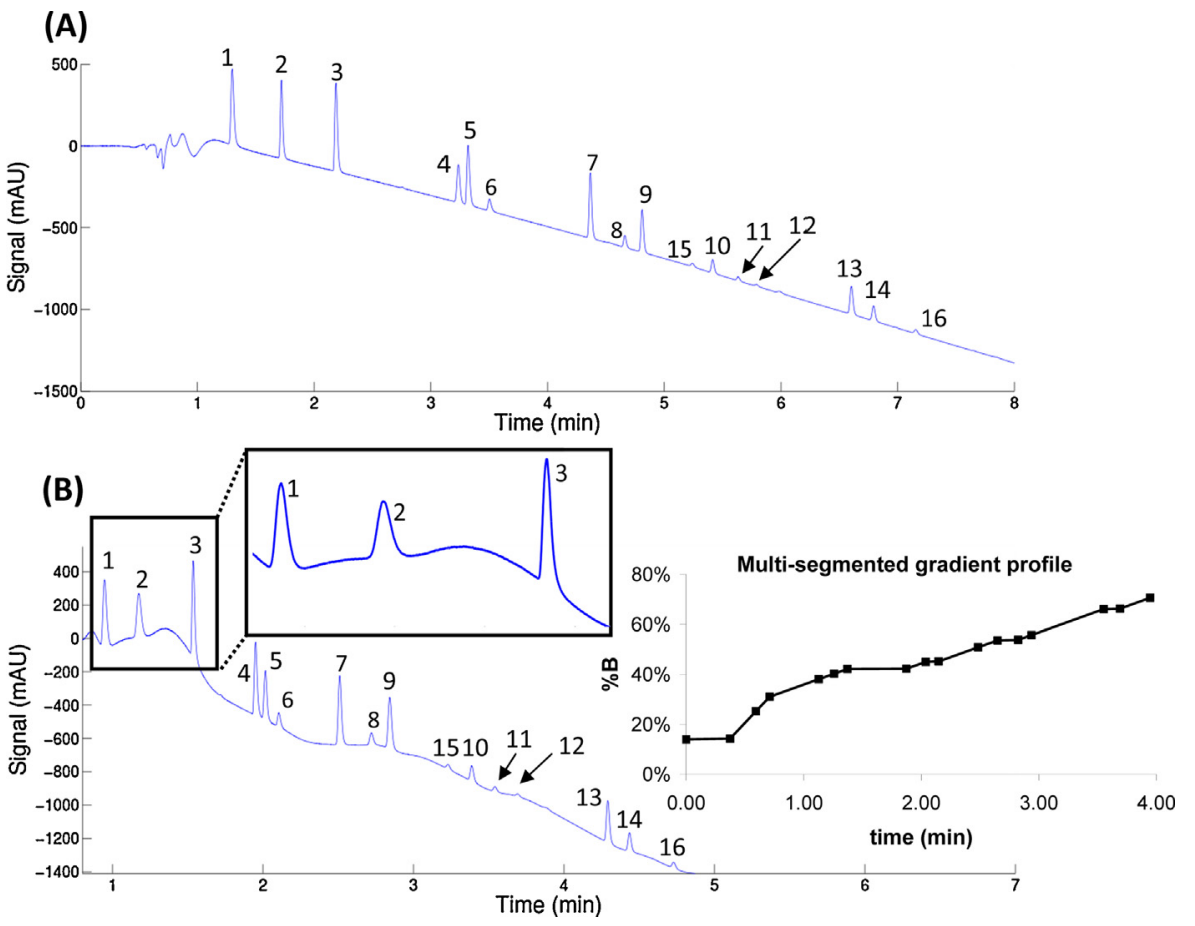

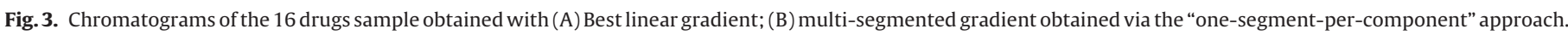

multi-segmented gradient obtained with the presently proposed "one-segment-per-component" approach. Moreover the resolution of the critical pair was worse (Fig. 2C).

\subsubsection{Sample 2: API and 13 impurities}

The second sample consists of an active pharmaceutical ingredient (API) and 13 degradation products. The "one-segmentper-component" optimization approach approximately results in a 3-segment gradient which could reduce the analysis time with about 50\% compared to the best simulated linear gradient (Fig. $\mathrm{S}-1 \mathrm{~A}$ and $\mathrm{B}$ ). In this case the "one-segment-per-component" strategy did not improve the $R_{S}$ or the analysis time compared to the multi-segmented gradient obtained with the commercial software package, using the multi-segment optimization mode (Fig. S-1C). The gradient conditions are given in Table $\mathrm{S}-3$. Because the compounds of the pharmaceutical sample are structurally closely related, the retention parameters of the Neue-Kuss model (cf. Eq. (1)), were determined using six instead of three scouting runs, including different gradient lengths $(5,10,15 \mathrm{~min})$ and different starting compositions ( $5 \%$ and $20 \%$ ), resulting in an average deviation between predicted and experimental retention times of $0.6 \%$ including the first eluting compounds (Table S-4). The exact modeling of the critical pair was found to be of uttermost importance as both peaks can switch elution order when changing the gradient slope.

\subsubsection{Sample 3: 16 drugs commonly found in waste water}

The third sample consists of 16 drugs that are commonly found in waste water [31]. The "one-segment-per-component" gradient includes an isocratic step at the beginning, followed by six linear segments that actually follow a curved (\%B,time)-pattern, starting with a steep (second) gradient segment to an almost isocratic step (7th gradient step) (Fig. 3). This curvature could again be approximated by only three linear segments, and different segments with similar slopes, which could be approximated by one linear segment. Gradient segments 8 to 16 follow an almost linear behavior, including three small isocratic steps (step 9,12 and 15). The "onesegment-per-component" optimization approach could reduce the analysis time with 35\% compared to the best linear gradient. The gradient conditions are given in Table S-5. The average deviation between the predicted and the experimental retention times was $2.4 \%$ (except for the first 2 eluting compounds, see Table 3 ). It was verified that although the baseline is quite complex, following the complex multi-segmented gradient program, the peaks can still be integrated accurately (see zoom-in in Fig. 3).

\subsubsection{Sample 4: 14 drugs commonly found in waste water}

For the subset of 14 components (numbers 1 to 14 from Sample 3 ) the analysis time could be reduced even more. Since the critical pair is the same as for the 16 component sample, the best linear gradient (9.5-75\% in $7.5 \mathrm{~min})$ is the same as for the 16 component mixture. On the other hand, the "one-segment-per-component" gradient approach (Table S-7), which now includes steeper slopes than was the case for sample 3, allowed to reduce the retention time of the last compound with another $1.2 \mathrm{~min}$. This demonstrates the flexibility of the proposed optimization strategy. The total

Table 3

Predicted and experimental retention times for Sample 3 (16 drugs commonly found in waste water) obtained via the "one-segment-per-component" approach.

\begin{tabular}{lll}
\hline$t_{R, \text { predicted }}$ & $t_{R, \exp }$ & Deviation $(\%)$ \\
\hline 1.18 & 0.95 & -9.9 \\
1.40 & 1.17 & -6.3 \\
1.52 & 1.54 & 1.1 \\
1.94 & 1.95 & 0.7 \\
2.07 & 2.02 & -2.5 \\
2.18 & 2.11 & -3.4 \\
2.68 & 2.51 & -6.4 \\
2.85 & 2.72 & -4.4 \\
2.96 & 2.84 & -3.9 \\
3.29 & 3.23 & -1.9 \\
3.46 & 3.39 & -2.0 \\
3.63 & 3.54 & -2.5 \\
3.75 & 3.69 & -1.5 \\
4.36 & 4.29 & -1.6 \\
4.50 & 4.44 & -1.4 \\
4.76 & 4.73 & -0.6
\end{tabular}



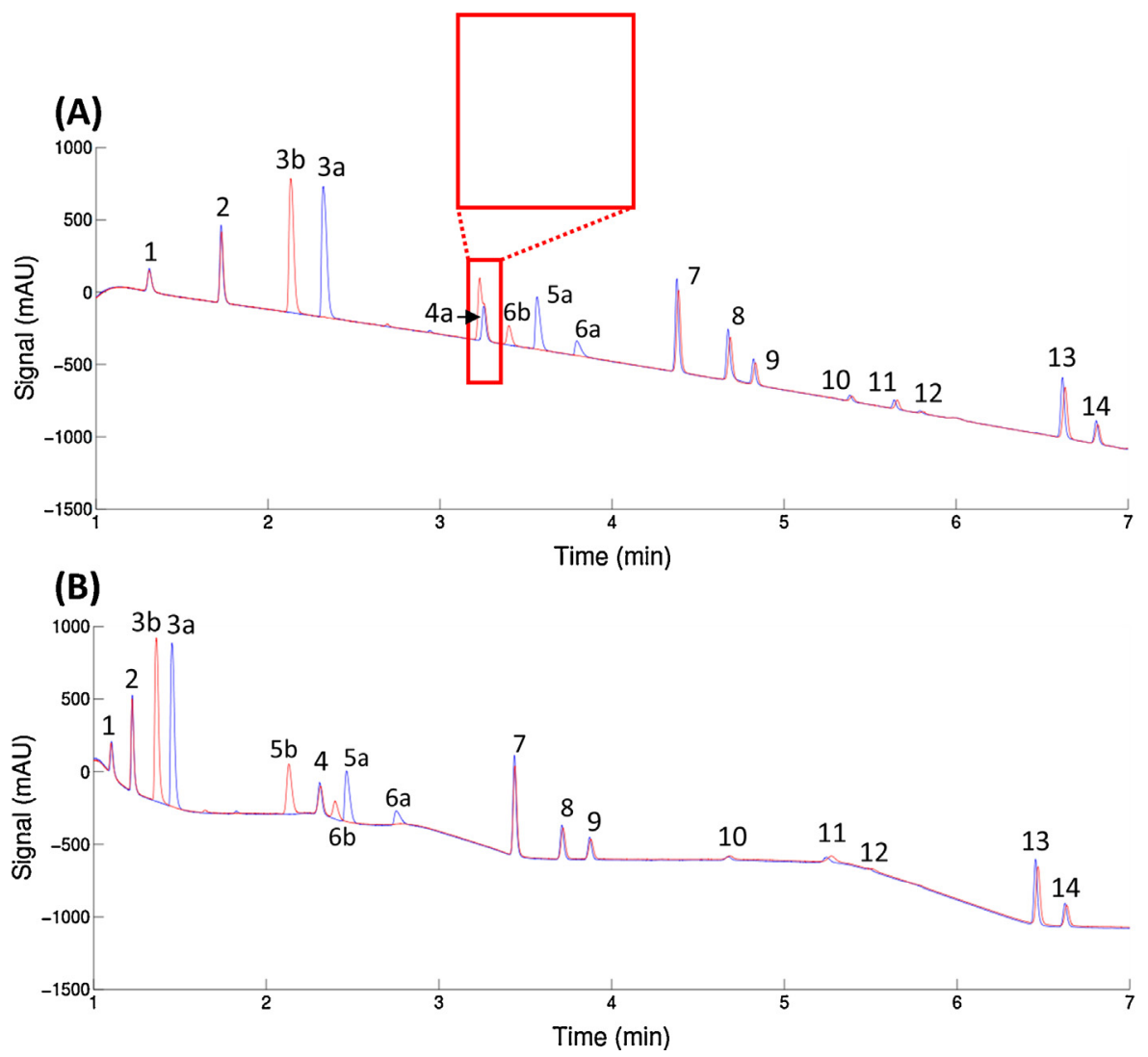

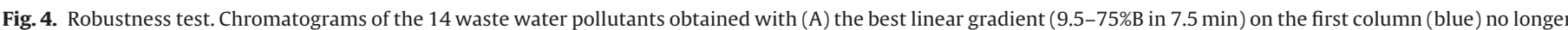

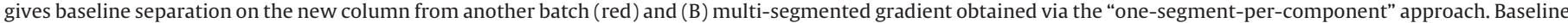

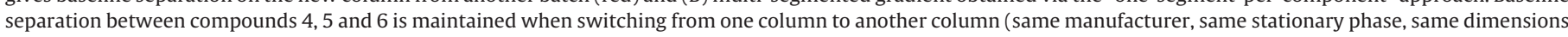
and particle size, but from a different batch). (For interpretation of the references to color in this figure legend, the reader is referred to the web version of this article.)

reduction in analysis time compared to the best linear gradient was in this case $48 \%$ (Fig. S-2). The average deviation between the predicted and the experimental retention times was $2.9 \%$ (Table S-6). It should be noted that in this case the pumping system is pushed to its very limits. Because the steep slope of the third segment (at low $\% \mathrm{ACN}$ ) leads to an overshoot in the baseline, the second and the third peak are in this case no longer be perfectly integrated (see zoom in Fig. S-2).

\subsection{Robustness test}

We also investigated whether the "one-segment-percomponent" approach leads to more robust gradient methods, i.e., less sensitive to small selectivity differences between different columns of the same type compared to the more conventional linear gradient optimization, because the "one-segment-percomponent" approach allows spacing the different peaks more equally over the entire chromatogram. Although perfect even spacing is of course the ultimate (unrealistic) goal and will indeed most of the time not be reached, even with a very complex gradient profile, the proposed one-segment-per-component gradient can be used to induce a more even spacing than in the case of a simple linear gradient and can in these cases be used as a way to increase the robustness of a separation when changing columns.

Numerically this is imposed by leaving out the maximum value for $R_{s, \text { crit }}$ while keeping the "if-then-else" construct to put an upper limit on the maximal analysis time. In this way, the peaks of the critical pair get some freedom to move while solutions leading to an excessively large spacing between the peaks are avoided because of the time constraint. This approach has been applied to the 14 component mixture (Sample 4 ), taking $t_{R \text {,max }}$ equal to the value of $t_{R, \text { last }}$ of the best linear gradient.

The robustness was tested experimentally by repeating the optimized linear and multi-segmented gradient on a new column from the same manufacturer, with the same stationary phase, column dimensions and particle size but from a different batch. As can be noted from Fig. 4A, switching from one column to another (although from the same type and the same manufacturer) dramatically reduces the resolution of the critical pair for the best linear gradient. On the other hand, the one-segment-per-component gradient program (Table S-9) resulted in baseline separated peaks on both columns (Fig. 4B), because of the much more even spacing of the peaks that can be achieved. The average deviation between the predicted and the experimental retention times was $1.4 \%$ except for the first eluting compound (Table S-8).

\section{Conclusion}

We developed a "one-segment-per-group-of-components" gradient design strategy and applied it to find the best possible multi-segment gradient program. The different strategies were applied to real (tar oil degradation products, pharmaceuticals) as well as to in silico samples. The analysis times were reduced significantly compared to the best single linear gradients. To account for the non-LSS behavior of the compounds, the model proposed by Neue and Kuss [23] was used to obtain the necessary accurate retention time predictions. Since the "one-segment-percomponent" method intrinsically allows to put the peaks in the chromatograms more closer to each other than the conventional gradient methods, our experience with the optimization of the 
experimental examples showed that any gain in prediction accuracy, even though it is only by $1 \%$, is very significant.

The newly proposed approach performed equally well or better than the multi-segment optimization mode of the commercial software package. The in silico study demonstrated the enhanced selectivity of the "one-segment-per-component" (10 component samples) and the "one-segment-per-two-components" $(20$ component samples) gradient design strategies compared to the traditional 4-segment grid search over a statistically significant amount of different samples (463 in total). Another advantage of the "one-segment-per-group-of-components" strategy is that the time needed to find the optimal gradient profile is much shorter than when optimizing the traditional multi-step gradient. This originates from the fact that the trajectory of the gradient program in the "one-segment-per-group-of-components" approach is auto-guided by the elution properties of the sample compounds. Hence, no time is wasted to searching in areas of the parameter space where there is anyhow no chance of finding a good solution.

Conceptually, the presently proposed segmented gradient optimization methods can readily fit in the advanced 3D or 4D MD strategies used nowadays [31,32]. either as the final step after selection of the most promising stationary phase, modifier solvent, $\mathrm{pH}$ and $\mathrm{T}$ (typically done via a design of experiments approach), or as an integral part of the 3D or 4D search algorithm. To achieve the latter, the $\mathrm{pH}$ - and T-dependency (and possibly also that of the stationary phase and type of organic modifier) should be modeled via a design of experiments, similar to what is done in current gradient optimization software. This modeled dependency can then be used in the search for the best one-component-per-segment gradient among all possible combinations of $\mathrm{T}$ and $\mathrm{pH}$ (and possibly also stationary phase and type of organic modifier). In this type of applications, the enhanced search speed of the one-componentper-segment method will prove its value even more, because now much more cases need to be considered for gradient optimization and it has been shown in the present study that the "one-segmentper-component" is at least one order of magnitude faster than the traditional multi-step gradient search.

Since it was also demonstrated the concept not only works by having one compound eluting per segment ("one-segment-percomponent") but also by grouping them in pairs, cf. the "onesegment-per-two-components" approach, many other variants can be conceived of (grouping in triplets, varying the number of compounds per segment, ....). Given the very short required search times, these different variants can furthermore be combined by running multiple consecutive searches to also optimize the grouping of the components. The robustness example given for the 14 component pharmaceutical mix sample also shows that, because of the increased possibility to space the peaks more evenly compared to traditional single and multi-step gradient methods, the "one-segment-per-component" gradient design algorithm can also be tuned to search for intrinsically more robust methods.

To minimize method transfer problems caused by potential instrument-depending "rounding" effects of the many individual gradient segments, especially when using older equipment, one could also consider implementing a fitting procedure to reduce the number of gradient segments, by retrofitting the profile obtained via the "one-segment-per-component" strategy with the best fitting 2,3, 4 or 5-segment profile. In this way, the rapid search offered by the "one-segment-per-group-of-components"-approach can be combined with the more robust "traditional" multi-step gradient profile with a pre-set maximum number of segments, which can also be more easily validated. In this way, the present procedure could still be beneficial for the separation of challenging mixtures for which multi-segment gradients can provide some additional selectivity, even with the use of older equipment.

\section{Acknowledgment}

The authors acknowledge financial support of the Research Foundation-Flanders (FWO-Vlaanderen). E.T. is the recipient of a Ph.D. fellowship of the FWO-Vlaanderen.

\section{Appendix A. Supplementary data}

Supplementary material related to this article can be found, in the online version, at http://dx.doi.org/10.1016/j.chroma. 2014.06.097.

\section{References}

[1] A. de Villiers, M. Kalili, M. Malan, J. Roodman, Improving HPLC separations of polyphenols, LC-GC Eur. 23 (2010) 466-478.

[2] P. Jandera, Can theory of gradient liquid chromatography be useful in solving practical problems? J. Chromatogr. A 1126 (2006) 195-218.

[3] J.W. Dolan, Selectivity in reversed-phase LC separations (Part 1): Solvent-type selectivity, LC-GC Eur. 23 (2010) 581-584.

[4] J.W. Dolan, Selectivity in reversed-phase LC separations (Part 2): Solventstrength selectivity, LC-GC Eur. 24 (2011) 20-24.

[5] M. Pursh, A. Scheiwer-Theobaldt, H. Cortes, A. Gratzfeld-Huesgen, H. Schulenberg-Shell, B.-W. Hoffmann, Fast, ultra-fast and high-resolution LC for separation of small molecules, oligomers and polymers, LC-GC Eur. 21 (2008) $152-159$

[6] M. De Beer, F. Lynen, K. Chen, P. Ferguson, M. Hanna-Brown, P. Sandra, Stationary-phase optimized selectivity liquid chromatography: development of a linear gradient prediction algorithm, Anal. Chem. 82 (2010) 1733-1743.

[7] S. Goga, S. Heinisch, J.L. Rocca, Retention and column efficiency in reversed phase liquid chromatography as a function of $\mathrm{pH}$ for optimization purposes, Chromatographia 48 (1998) 237-244.

[8] P. Nikitas, A. Papa-Louisi, New Approaches to linear gradient elution used for optimization in reversed-phase liquid chromatography, J. Liq. Chromatogr. Relat. Technol. 32 (2009) 1527-1576.

[9] Y. Dharmadi, R. Gonzalez, A better global resolution function and a novel iterative stochastic search method for optimization of high-performance liquid chromatographic separation, J. Chromatogr. A 1070 (2005) 89-101.

[10] J.M. Giddings, J.C. Davis, Statistical method for estimation of number of components from single complex chromatograms: theory, computer-based testing, and analysis of errors, Anal. Chem. 57 (1985) 2168-2177.

[11] T. Jupille, L. Snyder, I. Molnar, Optimizing multi-linear gradients in HPLC, LC-GC Eur. (2002) 596-601.

[12] V. Concha-Herrara, G. Vivó-Truyols, J.R. Torres-Lapasio, M.C. García-AlvarezCoque, Limits of multi-linear gradient optimization in reversed-phase liquid chromatography, J. Chromatogr. A 1063 (2005) 79-88.

[13] H. Xiao, X. Liang, P. Lu, Total analytical method for Radix astragali extract using two-binary multi-segment gradient elution liquid chromatography, J. Sep. Sci. 24 (2001) 186-196

[14] P. Nikitas, A. Pappa-Louisi, K. Papachristos, Optimisation technique for stepwise gradient elution in reversed-phase liquid chromatography, J. Chromatogr. A 1033 (2004) 283-289.

[15] M. De Beer, F. Lynen, M. Hanna-Brown, P. Sandra, Multiple step gradient analysis in stationary phase optimised selectivity LC for the analysis of complex mixtures, Chromatographia 69 (2009) 609-614.

[16] P. Jandera, J. Churacek, Gradient elution in column liquid chromatography: theory and practice, J. Chromatogr. 170 (1979) 1-10.

[17] Y. Shan, Z. Weibing, A. Seidel-Morgenstern, R. Zhao, Y. Zhang, Multi-segment linear gradient optimization strategy based on resolution map in HPLC, Sci. China Ser. B: Chem. 46 (2006) 315-325.

[18] M. Lämmerhofer, P.D. Di Eugenio, I. Molnar, W. Lindner, Computerized optimization of the high-performance liquid chromatographic enantioseparation of a mixture of 4-dinitrophenyl amino acids on a quinine carbamate-type chiral stationary phase using Drylab, J. Chromatogr. B 689 (1997) 123-135.

[19] E.F. Hewitt, P. Lukulay, S. Galushko, Implementation of a rapid and automated high performance liquid chromatography method development strategy for pharmaceutical drug candidates, J. Chromatogr. A 1107 (2006) 79-87.

[20] P. Nikitas, A. Pappa-Louisi, P. Agrafiotou, Multilinear gradient elution optimisation in reversed-phase liquid chromatography using genetic algorithms, J. Chromatogr. A 1120 (2006) 299-307.

[21] M.R. Hadjmohammadi, K. Kamel, Multi-linear gradient elution optimization for separation of phenylthiohydantoin amino acids using pareto optimality method, J. Iran. Chem. Soc. 7 (2010) 107-113.

[22] U.D. Neue, H-J. Kuss, Improved reversed-phase gradient retention modeling, J. Chromatogr. A 1217 (2010) 3794-3803.

[23] L.R. Snyder, Linear elution adsorption chromatography: VII. Gradient elution theory, J. Chromatogr. 11 (1964) 415-434

[24] L.R. Snyder, J.W. Dolan, High-performance Gradient Elution: The Practical Application of the Linear Solvent Strength Model, Willey Interscience, Hoboken, NJ, 2007. 
[25] P. Nikitas, A. Pappa-Louisi, Expressions of the fundamental equation of gradient elution and a numerical solution of these equations under any gradient profile, Anal. Chem. 77 (2005) 5670-5677.

[26] J.W. Dolan, D.C. Lommen, L.R. Snyder, Drylab computer simulation for high-performance liquid chromatographic method development: II. Gradient elution, J. Chromatogr. 485 (1989) 91-112.

[27] A.P. Schellinger, P.W. Carr, Isocratic and gradient elution chromatography: a comparison in terms of speed, retention reproducibility and quantitation, J. Chromatogr. A 1109 (2006) 253-266.

[28] S.L. Morgan, S.N. Deming, Optimization strategies for the development of gas-liquid chromatographic methods, J. Chromatogr. 112 (1975) 267-285.
[29] U.D. Neue, Theory of peak capacity in gradient elution, J. Chromatogr. A 1079 (2005) 153-161.

[30] D. Cabooter, D. Clicq, F. De Boever, F. Lestremau, R. Szucs, G. Desmet, A variable column length strategy to expedite method development, Anal. Chem. 83 (2011) 66-975.

[31] R. Komány, I. Molnár, H-J. Rieger, Exploring better column selectivity choices in ultra-high performance liquid chromatography using quality by design principles, J. Pharm. Biomed. 80 (2013) 79-88.

[32] I. Molnár, H-J. Rieger, K.E. Monks, Aspects of the design space in high pressure liquid chromatography method development, J. Chromatogr. A 1217 (2010) 3193-3200. 